\title{
A Bond-Graph Method for Flatness-Based Dynamic Feedback Linearization Controller Synthesis: Application to a Current-Fed Induction Motor
}

\author{
Ali Achir ${ }^{\dagger}$, Sergio Junco ${ }^{\S}$, Alejandro Donaire ${ }^{\S \ddagger}$ and Christophe Sueur ${ }^{\dagger}$ \\ $\dagger$ Laboratoire d'Automatique, Génie Informatique \& Signal, CNRS UMR 8146, Ecole Centrale de Lille, France \\ \{achir.ali, christophe.sueur\}@ec-lille.fr \\ $\S$ Departamento de Electrónica, Facultad de Ingeniería, Universidad Nacional de Rosario, Argentina. \\ † Consejo Nacional de Investigaciones Científicas y Técnicas - CONICET \\ \{adonaire,sjunco\}@fceia.unr,edu.ar
}

\begin{abstract}
KEYWORDS
Bond Graphs, Induction Motor, Differential Flatness, Exact Linearization and Decoupling.
\end{abstract}

\begin{abstract}
This paper presents a method for controller synthesis in the bond graph domain. A dynamic feedback linearization and decoupling controller of rotor speed and rotor-flux amplitude of a current-fed induction motor is derived on the basis of a flatness analysis entirely conducted on a two-input nonlinear bond graph model of the motor. Once the rotor speed has been found as the first flat output, a technique which uses a variational bond graph and its associated quotient bond graph (modulo the differential of the first flat output) allows identifying the second flat output as being the angle of the rotor flux-linkage space vector. The flat output parameterization of the control outputs is later used to derive the control law. Simulation results are given to demonstrate the control system performance.
\end{abstract}

\section{INTRODUCTION}

The main concern of this paper is a methodological approach on bond graphs (BG) to the development of control laws for nonlinear physical systems, thereby linking theoretical methods of nonlinear control with physically based modeling formalisms.

Some available results for multivariable models, based on differential geometry theory, exploit the notion of relative degree for the input-output decoupling, disturbance rejection and exact linearization problems. Nevertheless, these techniques which have a nice graphical (BG) interpretation in the linear case (Bertrand et al. 1997), cannot easily extended to nonlinear BGs. Moreover, they are restricted to systems without unstable zero dynamics.

A relatively recent and elegant technique that solves complex control problems is based on the property of flatness introduced in (Fliess et al. 1995). This technique, that enables to overcome the problem of unstable zero dynamics and to solve trajectory planning and tracking problems, has shown itself well suited to be performed on graphical models of dynamic systems. The most effective method to analyze the flatness property is based on the freeness of the tangent (variational) module associated with the nonlinear model. Indeed, in most cases the flatness property itself and the differential parameterization of the system variables by the flat outputs are not easily pointed out directly on the nonlinear system model. A variational approach introduced in a previous work for nonlinear BG models (Achir and Sueur 2005) enables to identify the flat outputs of non linear systems. Although the proposed algorithm is easily applicable in case of single input BGs, it comes up against the problem of computing the Kronecker indices in the multi input case. Nevertheless, it was shown that many BG tools developed for linear systems are no longer valid due to the fact that the model coefficients are time dependent and therefore new rules dealing with such problems have been proposed.

In this paper, the synthesis on the BG domain of a dynamic feedback linearization and decoupling controller of rotor speed and rotor-flux amplitude of a current-fed induction motor (IM) is proposed. As this is a multivariable (two-input, two-output) application of flatness, the method of the quotient BG is introduced in order to help obtaining the second flat output once the first flat output variable has been guessed on the base of physical grounds. This technique is an adaptation to the BG domain of a previous result (Fossas et al. 1998).

The article is organised as follows: Second Section presents a BG model of the current-fed IM which is well adapted to perform the flatness analysis that follows in the same section. Third Section addresses the controller synthesis of the IM. Next, Fourth Section presents some simulation results demonstrating the closed-loop performance of the control system. The conclusions are presented in Fifth Section. Some mathematical background on flatness, the concept of ring $\mathrm{BG}$ are recalled in the Appendix.

\section{FLATNESS ANALYSIS ON A BG MODEL OF THE IM}

\section{BG Model of Current-Fed Induction Motor}

A BG model of a current-fed induction motor convenient for the purposes of this paper is given in Fig. 1 . It is set forth in a stationary (or stator fixed) reference frame with orthogonal axes $d$ and $q$. The controlled flow sources impress the stator currents $i_{s d, q}$. The energy 
variables associated to both generalized inertia components $I: L_{r}$ are $\Phi_{r d, q}$, the rotor flux linkage components. The inertia $I: J$ is related to the angular momentum $J \omega$; linear friction with coefficient $b$ is assumed. The coefficients $M$ and $L_{r}$ denote the mutual and rotor self-inductance, respectively; $R_{r}$ is the rotor resistance, and $p$ the number of pole-pairs.

Applying standard BG equation-reading procedures (Karnopp et al. 1990) on each of the three 1-junctions associated to the mentioned energy storages, this BG yields the set of voltage and mechanical balance equations (1) and (2), respectively. Note that the driving torque (3) is exclusively supplied by the diagonalplaced MGYs (moduli $p M \Phi_{r x} / L_{r}$ ) on the left of the BG, while both $\boldsymbol{M G Y S}$ on the right (moduli $p \Phi_{r x}$ ) provide a zero net effort on the mechanical 1 -junction. Equations (4) and (5) relate the pair of BG-currents $\left(i_{d}, i_{q}\right)$ to $\left(i_{s d}\right.$, $\left.i_{s q}\right)$ and $\left(i_{r d}, i_{r q}\right)$, the stator and rotor currents, respectively.

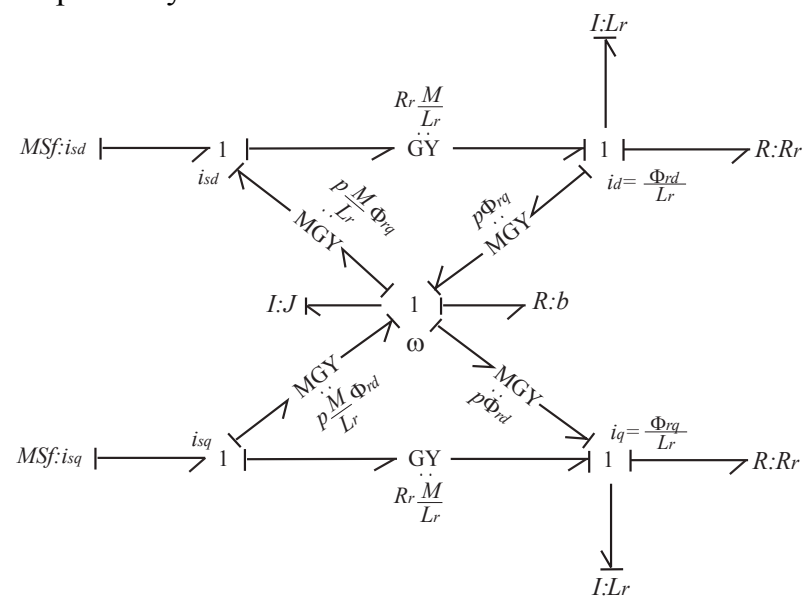

Fig. 1. $d$ - $q$ reference-frame BG-model of current-fed IM

$$
\begin{aligned}
& \left\{\begin{array}{l}
\dot{\phi}_{r d}+R_{r} i_{d}-M \frac{R_{r}}{L_{r}} i_{s d}+p \omega \phi_{r q}=0 \\
\dot{\phi}_{r q}+R_{r} i_{q}-M \frac{R_{r}}{L_{r}} i_{s q}-p \omega \phi_{r d}=0
\end{array}\right. \\
& p M\left(\phi_{r d} i_{s q}-\phi_{r q} i_{s d}\right) / L_{r}-J \dot{\omega}-b \omega=0 \\
& T_{e m}=p M\left(\phi_{r d} i_{s q}-\phi_{r q} i_{s d}\right) / L_{r} \\
& i_{d}=\phi_{r d} / L_{r}=M i_{s d} / L_{r}+i_{r d} \\
& i_{q}=\phi_{r q} / L_{r}=M i_{s q} / L_{r}+i_{r q}
\end{aligned}
$$

\section{Flat Outputs Determination on the BG Model}

As the system has two control inputs, two flat outputs are to be determined. This task is performed on the BG model applying the four-step procedure reported in the Appendix.

1) Variational Bond Graph: Build a Tangent or Variational BG (VBG) via differentiation of the original nonlinear BG.

2) Quotient $V B G(Q V B G)$ : Guess a trivial basis of the VBG (a differential 1-form) and quotient the VBG modulo this basis (two-input model reduced to a singleinput one).
3) QVBG Basis: Identify the basis (a second differential 1-form) of the $Q V B G$.

4) Integration: Integrate both differential 1-forms to find the flat outputs of the original nonlinear BG.

The application of this procedure will show next that the rotor speed and the angle of the rotor-flux-linkage space-vector (or the rotor flux angle, for short) are the two flat outputs looked-for.

\section{1) Constructing the $V B G$}

Applying the Kähler differential operator (see Appendix) to the nonlinear BG of Fig. 1 yields the VBG, which is built by simply connecting the VBGimages of the original $\mathrm{BG}$ components, due to the linearity of the Kähler operator. The only images to be determined are those corresponding to the nonlinear components, i.e., the modulated gyrators. To deal with, consider the modulated gyrator depicted in Fig. 2. Its constitutive equations are given in (6). Their differentials are given in (7), where $m$ is a nonlinear function depending on the state variables and even on the input variables in general case.

$$
1 \longmapsto \frac{e_{1}}{f_{1}} \stackrel{m}{M} \dot{G} Y \frac{e_{2}}{f_{2}}{ }_{1} 1
$$

Fig. 2. Modulated gyrator

$$
\begin{gathered}
e_{2}=m f_{1} \quad, \quad e_{1}=m f_{2} . \\
d e_{2}=m d f_{1}+f_{1} d m \\
d e_{1}=m d f_{2}+f_{2} d m
\end{gathered}
$$

These equations suggest building the VBG by augmenting the initial modulated gyrator with two effort sources as shown in Fig. 3. Therefore, the VBG of the current-fed IM is built as shown in Fig. 4.

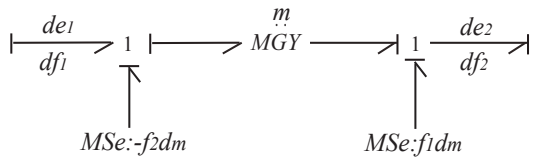

Fig. 3: VBG of a modulated Gyrator

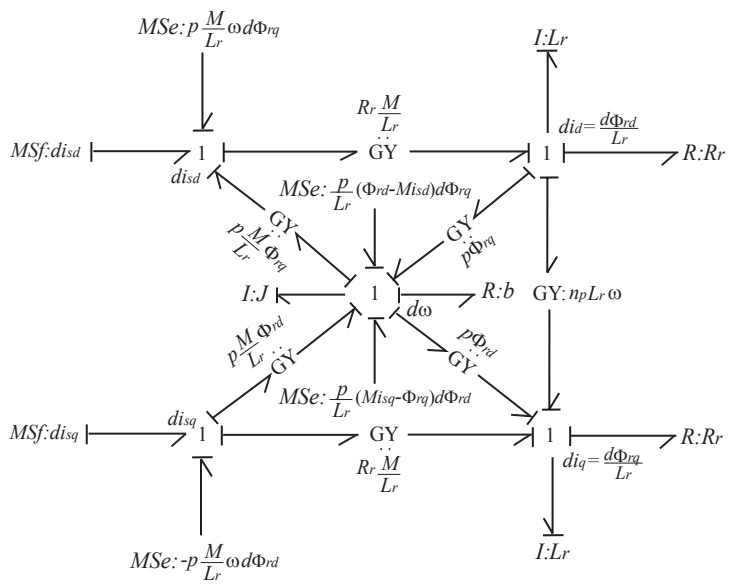

Fig. 4. VBG of current-fed IM

In the sequel, the following generalized notation will be used for the input and state variables, according to the 
standard usage in control theory:

$$
\begin{aligned}
& d u=\left(d u_{1}, d u_{2}\right)^{t}=\left(d i_{s d}, d i_{s q}\right)^{t} \\
& d x=\left(d x_{1}, d x_{2}, d x_{3}\right)^{t}=\left(d i_{d}, d i_{q}, d \omega\right)^{t}
\end{aligned}
$$

\section{2) Constructing the $Q V B G$}

The VBG of Fig. 4 represents the module $\Omega$ modeling the tangent system. An obvious choice of a basis is the mechanical speed $d y_{1}=d x_{3}$. In order to get the second basis, a quotient modulo $d y_{1}$ is made, which results in the new module $\Omega_{1}=\Omega /\left\langle d y_{1}\right\rangle$. Performing this quotient operation is equivalent to setting $d x_{3}=d \dot{x}_{3}=0$. This means that the flow and the effort in the BG-elements $I: J$ and $R: b$ are equal to zero. By removing them, the 1 -junction associated to $d x_{3}$ gives the algebraic relation (9) between $d \bar{u}_{1}=d \bar{i}_{s d}$ and $d \bar{u}_{2}$ $=d \bar{i}_{s q}$ (the bars denoting variables in the quotient space).

$$
\phi_{r q} d{\overline{i_{s d}}}_{-\phi_{r d}} d{\overline{i_{s q}}}+i_{s d} d \bar{\phi}_{r q}-i_{s q} d \bar{\phi}_{r d}=0
$$

Solving (9) for $d \bar{u}_{2}$ and replacing $d \bar{u}_{2}$ in the VBG of Fig. 4 leads to the QVBG of Fig. 5.

\section{3) Calculating the basis of the QVBG model}

The QVBG just obtained has only one single input source, and it is thus possible to apply the procedure given in the appendix to identify the second basis. Let it be

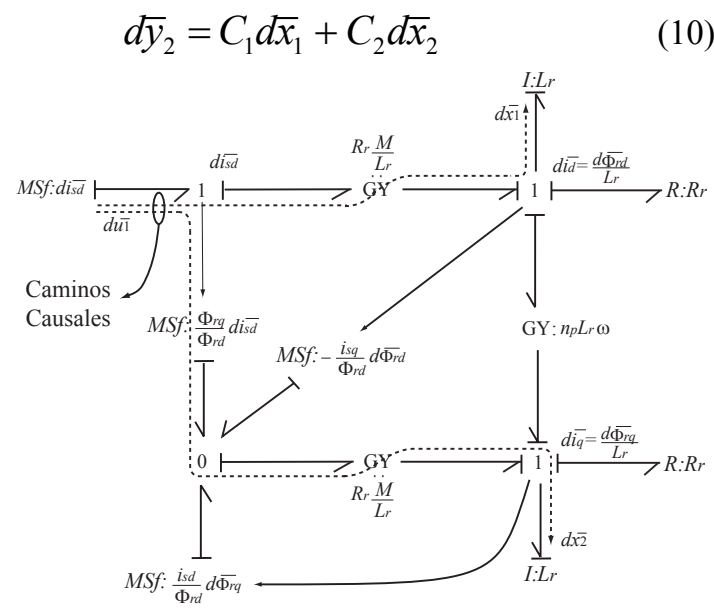

Fig. 5. Quotient BG model

$G_{l}\left(d \bar{u}_{1}, d \bar{x}_{i}\right)$ stands for the gain of the causal path of length one between the input $d \bar{u}_{1}$ and the storage having $d \bar{x}_{i}$ as state variable.

$$
C_{1} G_{1}\left(d \bar{u}_{1}, d \bar{x}_{1}\right)+C_{2} G_{1}\left(d \bar{u}_{1}, d \bar{x}_{2}\right)=0
$$

The values of these gains are easily calculated on the
QVBG of Fig. 6 as given in (12), (13). As regards the notation in (13): it has been set $x_{2} / x_{1}=i_{q} / i_{d}$ in accordance with the definitions (8.2). Indeed, note that the gain read on the QVBG is $M \Phi_{r q} / T_{r} \Phi_{r d}$, which equals that given in (13) because of the definitions (4).

$$
\begin{aligned}
& G_{1}\left(d \bar{u}_{1}, d \bar{x}_{1}\right)=M R_{r} / L_{r} \\
& G_{1}\left(d \bar{u}_{1}, d \bar{x}_{2}\right)=M R_{r} x_{2} / L_{r} x_{1}
\end{aligned}
$$

Choosing $C_{1}=x_{2}$ yields the solution $C_{2}=-x_{1}$. Therefore the basis of the module $\Omega_{1}$ is given by (14).

$$
d \bar{y}_{2}=x_{2} d \bar{x}_{1}-x_{1} d \bar{x}_{2}
$$

Consequently, the bases of $\Omega$ are as given in (15).

$$
\left\{\begin{array}{l}
d y_{1}=d x_{3} \\
d \bar{y}_{2}=x_{2} d \bar{x}_{1}-x_{1} d \bar{x}_{2}+p\left(\frac{d}{d t}\right) d x_{3}
\end{array}\right.
$$

\section{4) Integrating the Differential 1-forms}

The first 1-form is trivially integrable, and the second is integrable after multiplying it by the integrating factor $\left(x_{1}^{2}+x_{2}^{2}\right)^{-1}$ and setting the polynomial $p\left(\frac{d}{d t}\right)=0$. Finally, the flat outputs of the original nonlinear BG are given by (16). As anticipated when starting this section, they are the rotor speed $\omega$ and the rotor flux angle $\rho$.

$$
\left\{\begin{array}{l}
y_{1}=x_{3}=\omega \\
y_{2}=\tan ^{-1}\left(x_{2} / x_{1}\right)=\tan ^{-1}\left(\phi_{r q} / \phi_{r d}\right)=\rho
\end{array}\right.
$$

\section{Rotor-Flux Parameterization by the Flat Outputs}

It is well-known that all system variables can be differentially parameterized by the flat outputs. Next, the parameterization of the rotor-flux amplitude is calculated, what will be useful for the purposes of controller design.

The time-derivative of $y_{1}$, the first flat output, is just the rotor-speed state equation (17). On the other hand, (18) is the expression of the time-derivative of the second flat output $y_{2}$, with $\Delta=\left(\phi_{r d}^{2}+\phi_{r q}^{2}\right)=\psi_{r}^{2}$, the square of the rotor flux amplitude. Both equations can be immediately derived from Fig. 1 applying standard BG equation-reading procedures (Karnopp et al. 1990) (see next section). The second term in the right-hand side of (18) is the (dynamic) slip-speed between the speed of the rotor flux vector and the rotor speed, the latter being measured in electrical radians per second.

$$
\begin{aligned}
& \dot{y}_{1}=-\frac{b}{J} y_{1}+\frac{p M}{J L_{r}}\left(x_{1} u_{2}-x_{2} u_{1}\right) \\
& \dot{y}_{2}=p y_{1}+\frac{M}{T_{r}} \frac{\left(x_{1} u_{2}-x_{2} u_{1}\right)}{\Delta}
\end{aligned}
$$

Its is easy to derive (19) by elimination of the parenthesis $\left(\begin{array}{llll}x_{1} & u_{2}-x_{2} & u_{1}\end{array}\right)$ from (17) and (18). This equation entails the parameterization of the rotor flux amplitude by the two flat outputs. 


$$
\dot{y}_{2}=p y_{1}+\frac{b R_{r}}{p} \frac{y_{1}+(J / b) \dot{y}_{1}}{\psi_{r}^{2}}
$$

\section{CONTROLLER SYNTHESIS}

The flatness property enables to transform the system represented by the bond graph of Fig. 1 into a linear controllable one without zero dynamics by means of an endogenous dynamic feedback and change of coordinates. This can be achieved by applying the standard input-output decoupling algorithm, that involves differentiating the outputs until auxiliary inputs appear in a non singular way. This may require the intermediate addition of integrators on the input channels, which become states of the dynamic compensator.

After the synthesis of the dynamic feedback

linearization controller, a linear overriding controller is designed in order to solve a tracking task for both, the rotor speed and the amplitude of the rotor flux vector.

\section{Dynamic Feedback Linearization and Decoupling}

The design of this compensator is supported by the BGmodel of Fig. 1. The first derivative $\dot{y}_{1}=\dot{\omega}$ of the first flat output is obtained by evaluating the effort at the $I: J$ storage element using its associated 1 -junction equation.

$$
\dot{y}_{1}=-\frac{b}{J} \omega+p \frac{M}{J L_{r}}\left(\phi_{r d} i_{s q}-\phi_{r q} i_{s d}\right)
$$

The first derivative $\dot{y}_{2}$ of the second flat output is given in (20), with $\dot{\phi}_{r d}$ and $\dot{\phi}_{r q}$ as in (21). The latter equations are obtained by evaluating the efforts in the $I: L_{r}$ storages using the constitutive relationships of their associated 1 -junctions and following the causality rules.

$$
\begin{gathered}
\dot{y}_{2}=\frac{\phi_{r d}}{\Delta} \dot{\phi}_{r d}-\frac{\phi_{r q}}{\Delta} \dot{\phi}_{r q}, \\
\left\{\begin{array}{l}
\dot{\phi}_{r d}=-\frac{R_{r}}{L_{r}} \phi_{r d}+M \frac{R_{r}}{L_{r}} i_{s d}-p \omega \phi_{r q} \\
\dot{\phi}_{r q}=-\frac{R_{r}}{L_{r}} \phi_{r q}+M \frac{R_{r}}{L_{r}} i_{s q}+p \omega \phi_{r d}
\end{array}\right.
\end{gathered}
$$

Finally, replacing (21) in (20) yields (22), a slight variation of the anticipated equation (18).

$$
\dot{y}_{2}=\frac{1}{\Delta}\left(M \frac{R_{r}}{L_{r}}\left(\phi_{r d} i_{s q}-\phi_{r q} i_{s d}\right)+p \omega \Delta\right)
$$

The decoupling matrix is then

$$
G_{1}=\left[\begin{array}{cc}
-p \frac{M}{J L_{r}} \phi_{r q} & p \frac{M}{J L_{r}} \phi_{r d} \\
-\frac{M}{\Delta} \frac{R_{r}}{L_{r}} \phi_{r q} & \frac{M}{\Delta} \frac{R_{r}}{L_{r}} \phi_{r d}
\end{array}\right]
$$

This decoupling matrix $G_{1}$ is singular because $\operatorname{det}\left(G_{1}\right)=0$. Therefore, the flat outputs must be differentiated again. Setting $\xi=\left(\phi_{r d} i_{s q}-\phi_{r q} i_{s d}\right)$ and differentiating again the first flat output leads to (23), with $v_{1}=\dot{\xi}$.

$$
\ddot{y}_{1}=-\frac{b}{J}\left(-\frac{b}{J} \omega+p \frac{M}{J L_{r}} \xi\right)+p \frac{M}{J L_{r}} v_{1}
$$

Differentiating again the second flat output yields

$\ddot{y}_{2}=\frac{M}{\Delta} \frac{R_{r}}{L_{r}} v_{1}-M \frac{R_{r}}{L_{r}} \frac{\dot{\Delta}}{\Delta^{2}} \xi+p\left(-\frac{b}{J} \omega+p \frac{M}{J L_{r}} \xi\right)$,

with $\dot{\Delta}=-2 \frac{R_{r}}{L_{r}} \Delta+2 \frac{M R_{r}}{L_{r} \phi_{r d}}\left(\phi_{r q} \xi+\Delta i_{s d}\right)$,

Set $i_{s d}=v_{2}$, then

$$
\begin{aligned}
\ddot{y}_{2}=\frac{M}{\Delta} \frac{R_{r}}{L_{r}} v_{1}-\frac{M R_{r}}{L_{r}} \frac{\xi}{\Delta^{2}}\left[-2 \frac{R_{r}}{L_{r}} \Delta\right. & \left.+2 \frac{M}{\phi_{r d}} \frac{R_{r}}{L_{r}}\left(\phi_{r q} \xi+\Delta v_{2}\right)\right] \\
& +p\left(-\frac{b}{J} \omega+\frac{p M}{J L_{r}} \xi\right)
\end{aligned}
$$

The new decoupling matrix is regular

$$
G_{2}=\left[\begin{array}{cc}
\frac{p M}{J L_{r}} & 0 \\
\frac{M R_{r}}{\Delta L_{r}} & -2\left(\frac{M R_{r}}{L_{r}}\right)^{2} \frac{\xi}{\Delta \phi_{r d}}
\end{array}\right]
$$

thus, the control law can be calculated after (27).

$$
\left[\begin{array}{l}
v_{1} \\
v_{2}
\end{array}\right]=G^{-1}\left[\begin{array}{c}
w_{1}-\left(\frac{b}{J}\right)^{2} \omega+\frac{p b M}{J^{2} L_{r}} \xi \\
w_{2}-\frac{M}{L_{r}}\left(2 \frac{R_{r}^{2}}{\Delta L_{r}^{2}}+\frac{p^{2}}{J}\right) \xi+2\left(\frac{M R_{r}}{L_{r}}\right)^{2} \frac{\phi_{r q}}{\phi_{r d}} \frac{\xi^{2}}{\Delta^{2}}+p \frac{b}{J} \omega
\end{array}\right]
$$

The control laws for the original system are:

$$
\begin{gathered}
u_{1}=\frac{J L_{r}^{2} \phi_{r d}}{2 p M^{2} R_{r} \xi}\left(w_{1}-\left(\frac{b}{J}\right)^{2} \omega+\frac{b p M}{J^{2} L_{r}} \xi\right)-\frac{\Delta \phi_{r d}}{2 M^{2} R_{r}^{2} \xi}\left\{w_{2}\right. \\
\left.-\frac{M}{L_{r}}\left(2 \frac{R_{r}^{2}}{\Delta L_{r}^{2}}+\frac{p^{2}}{J}\right) \xi+2 M^{2} \frac{R_{r}^{2}}{L_{r}^{2}} \frac{\phi_{r q}}{\phi_{r d}} \frac{\xi^{2}}{\Delta^{2}}+p \frac{b}{J} \omega\right\} \\
u_{2}=\frac{\xi+x_{2} u_{1}}{x_{1}}
\end{gathered}
$$

With this controls the system is equivalent to a chain of 
two integrators

$$
\left\{\begin{array}{l}
\ddot{y}_{1}=w_{1} \\
\ddot{y}_{2}=w_{2}
\end{array},\right.
$$

with $w_{1}$ and $w_{2}$ the new input variables. The compensator dynamics is

$$
\dot{\xi}=\frac{J L_{r}}{p M}\left(w_{1}-\left(\frac{b}{J}\right)^{2} \omega+p \frac{b M}{J^{2} L r} \xi\right),
$$

The control laws are implemented as shown in Fig. 6 . Note that they are not defined for $\xi=0$ and $\phi_{r d}=0$. This can be remedied by adding a small constant value for the measured state $\phi_{r d}$ and a suitable constant value to the compensator state $\xi$.

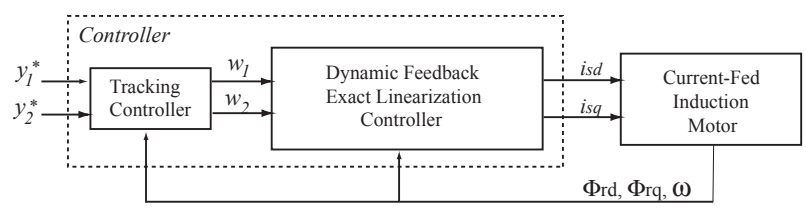

Fig. 6 Controller scheme

\section{Trajectory Tracking}

To ensure tracking of given references $y_{1}^{*}$ and $y_{2}^{*}$, one can define the reference errors $e_{1}=y_{1}^{*}-y_{1}$ and $e_{2}=y_{2}^{*}-y_{2}$, and prescribe the second order errors dynamics

$$
\left\{\begin{array}{c}
\ddot{e}_{1}+k_{11} \dot{e}_{1}+k_{12} e_{1}=0 \\
\ddot{e}_{2}+k_{21} \dot{e}_{1}+k_{22} e_{2}=0
\end{array},\right.
$$

where $k_{11}, k_{12}, k_{21}$, and $k_{22}$ are such that the tracking errors converge asymptotically to zero. Replacing these error expressions in (30), the control laws are obtained to be

$$
\left\{\begin{array}{c}
w_{1}=\ddot{y}_{1}+k_{11}\left(\dot{y}_{1}^{*}-\dot{y}_{1}\right)+k_{12}\left(y_{1}^{*}-y_{1}\right) \\
w_{2}=\ddot{y}_{2}+k_{21}\left(\dot{y}_{2}^{*}-\dot{y}_{2}\right)+k_{22}\left(y_{2}^{*}-y_{2}\right)
\end{array} .\right.
$$

Note that $y_{2}$ is not a control output per se, as is the case of $y_{1}$. In order to assure the tracking of a flux-amplitude reference $\psi_{r}^{*}$, the parameterization (19) is used under the form (34).

$$
\dot{y}_{2}^{*}=p y_{1}^{*}+\frac{b R_{r}}{p} \frac{y_{1}^{*}+(J / b) \dot{y}_{1}^{*}}{\psi_{r}^{* 2}}
$$

\section{SIMULATION RESULTS}

The closed loop control system has been simulated with the IM parameters given in Table I and the following values for the controller parameters: $k_{11}=20, k_{12}=100$, $k_{21}=200$, and $k_{22}=10,000$. A very good tracking performance is observed for the flat outputs as well as for the control outputs (Figs. 7-9). It can also be seen that the control inputs as well as the internal variables remain between admissible bounds for the machine at hands and behave smoothly (Figs. 10 and 11).
Table I. Induction Motor Parameters

\begin{tabular}{|l|l|}
\hline Rated Power & $17.65 \mathrm{Kw}$ \\
\hline Rated Speed & $1430 \mathrm{r} . \mathrm{p} . \mathrm{m}$ \\
\hline Rated Frequency & $50 \mathrm{~Hz}$ \\
\hline Rotor Resistance & $0.415 \Omega$ \\
\hline Rotor Self-Inductance & $87.62 \mathrm{mH}$ \\
\hline Mutual Inductance & $84.62 \mathrm{mH}$ \\
\hline Number of pole-pairs & 2 \\
\hline Load Torque Linear Coefficient & $0.7869 \mathrm{Nm} / \mathrm{s}$ \\
\hline Stator Self-Inductance & $86.94 \mathrm{mH}$ \\
\hline Stator Resistance & $0.371 \Omega$ \\
\hline Rated Voltage & $3 \times 380 \mathrm{v}$ \\
\hline
\end{tabular}

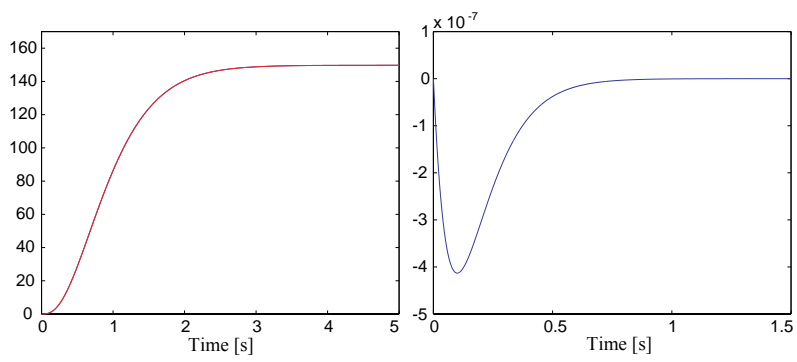

Fig. 7. a) Rotor speed $\omega$ and its reference $\omega^{*}$. b) Rotor speed error $\omega^{*}-\omega$.
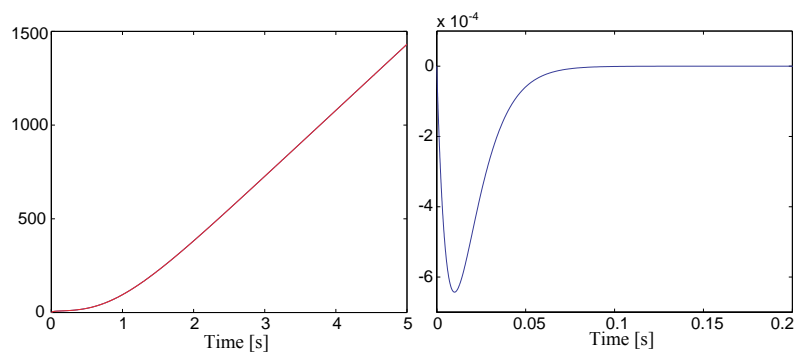

Fig. 8. a) Rotor flux angle and its reference. $b$ ) Rotor flux angle error.

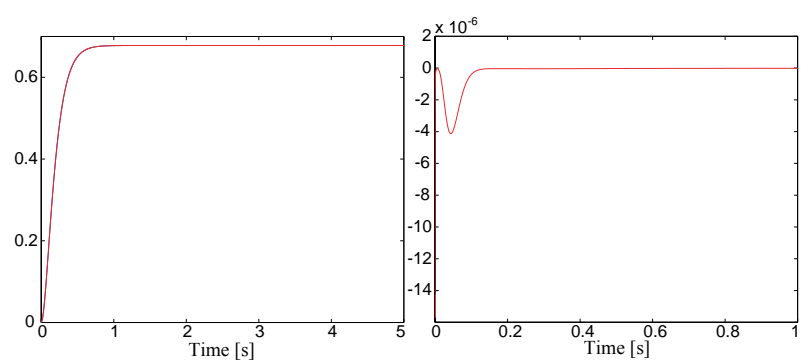

Fig. 9. a) Rotor flux amplitude $\psi_{r}$. b) Rotor flux amplitude error.

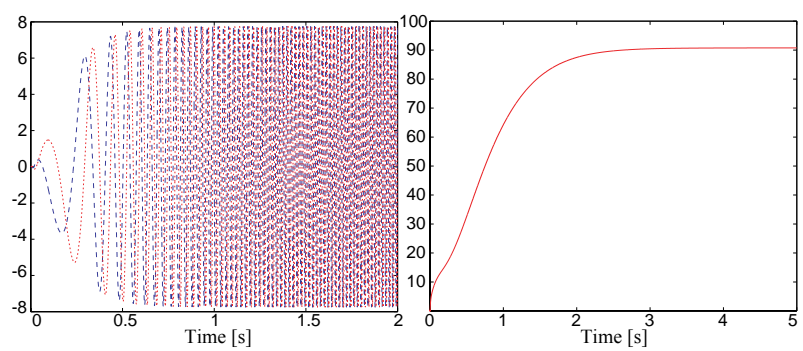

Fig. 10. a) State variables $x_{1}=i_{d}$ and $x_{2}=i_{q} . b$ ) Amplitude of stator current space vector. 


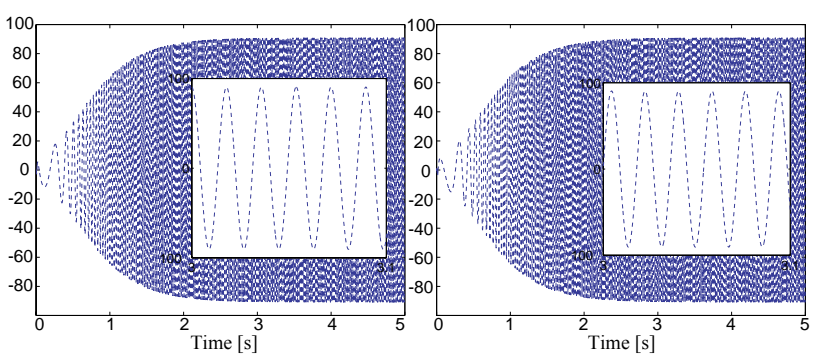

Fig. 11. a) Control input: stator current $i_{s d}$. b) Control input: stator current $i_{s q}$

\section{CONCLUSION}

A multivariable nonlinear tracking control problem has been solved via the application of a flatness-based method on the BG model of a current-fed induction machine. A technique for the systematic identification of the flat outputs has been addressed as part of the method. The work shows the feasibility of applying advanced and sophisticated nonlinear techniques on physically-based graphical models which are wellknown to modeling and simulation practicionists. Further work will address the derivation of the controller completely in the graphical domain using inversion techniques on bond graphs. Also of interest is extending the methodology to the more complex case of the voltage-source controlled induction machine, and to other kinds of physical plants. The later seems feasible (Achir, 2005), yet constructing the VBG, finding its flat outputs, and integrating them could be laborious for higher order, strongly nonlinear systems.

\section{ACKNOWLEDGMENT}

This work was in part supported by the ECOS-Sud Committee (France) and the Secretary of State STCIP (Argentina) under Grant No. A01E03 of the FrenchArgentine Scientific Cooperation Program.

\section{APPENDIX}

\section{Basic Definitions}

A brief recall on differential algebraic module theory as introduced in control theory in (Fliess et al. 1995, Fliess $1989,1990,1998)$ will help understanding the foundations of the method presented in this paper.

Assume $L$ and $K$ two differential fields. A non linear dynamic system is a finitely generated extension $L / K$, i.e.; there exists a finite set $\xi=\left(\xi_{1}, \ldots, \xi_{n}\right)$ in $L$ such that $L$ is differentially generated by $L$ and $\xi$.

Similarly, a linear system is a finitely generated left $K[d / d t]$-module $\Lambda$. A linear dynamics with input $u=\left(u_{1}, \ldots, u_{m}\right)$ is a linear system which contains $u$ such that the quotient module $\Lambda /[u]$ is torsion.

To a finitely generated field extension $L / K$ associate a mapping $d: L \rightarrow \Omega$ called Kähler differential and where $\Omega$ is a finitely generated left $L[d / d t]$-module.
The left $L[d / d t]$-module $\Omega$ is called the variational linear system associated with the system $L / K$.

\section{Flat Outputs Identification Procedure}

First, a procedure of identification of the flat outputs of non linear BG-models based on the notion of variational BG-model is recalled from (Achir et al. 2004), then a technique based on quotient of modules already presented in (Fossas et al. 1998) is applied to bond graphs.

Let $\dot{x}=f(x, u)$ be a state variable representation of a given non linear BG-model. $x$ and $u$ are the state and input variables associated with the dynamic elements and the input sources respectively. Applying Kähler differential operator, the variational BG-model is described by the following state variable representation

$$
\frac{d}{d t} d x=A(x, u) d x+B(x, u) d u
$$

where $A(x, u)=d f / d x, B(x, u)=d f / d u$ and $d x$ and $d u$ stands for the Kähler differentials of $x$ and $u$ respectively.

If its associated module is free, then the nonlinear model is strongly accessible (Fliess et al. 1995) and the nonlinear model is flat if the bases of $\Omega$ are integrable. Consider the following change of coordinates given by

$$
d x=P d z
$$

$P$ is the controllability matrix represented by (A. 3) $P=\left[B(x, u),(A(x, u)-d / d t) B(x, u), \ldots,(A(x, u)-d / d t)^{n-1} B(x, u)\right\rfloor$

The flat output is clearly the last variable $z_{n}$ of the considered controller form. In terms of state variables, the flat output take the following form:

$$
d y=\sum_{i=1}^{n} C_{i}(x) d x_{i}
$$

As a consequence of (A. 1-4), the row vector $C$ with entries $C_{i}$ satisfy the following set of algebraic equations

$$
\left\{\begin{array}{c}
C\left[A(x, u)-\frac{d}{d t}\right]^{k-1} B(x, u)=0 \quad k<n \\
C\left[A(x, u)-\frac{d}{d t}\right]^{n-1} B(x, u) \neq 0
\end{array}\right.
$$

The entries of the column vectors of the matrix $P$; i.e: $\left[A(x, u)-\frac{d}{d t}\right]^{(k-1)} B(x, u)$ are obtained from the variational bond graph model by means of the following formula:

$$
\left\{\left[A(x, u)-\frac{d}{d t}\right]^{k-1} B(x, u)\right\}_{i}=\sum_{H} G_{k}\left(d u, d x_{i}\right)
$$

$G_{k}\left(d u, d x_{i}\right)$ denotes the gain of the causal path of 
length $k$ connecting the input variable $d u$ to the state variable $d x_{i}$ and $H$ denotes the set of all the causal paths existing in the variational bond graph.

Remark: Due to the non commutative aspect, the gain of a causal path has to be calculated backward, i.e: from the output (sensor) variable to the input (source) variable.

In case of VBG-models with $m$ input sources, assume that $m-1$ flat outputs have already been guessed. In order to obtain the last flat output, a quotient of module is made. Let $\Omega_{m}$ be a module representation of the resulting $\mathrm{BG}$-model:

$$
\Omega_{m}=\frac{\Omega}{\left\langle d y_{1}, \ldots, d y_{m-1}\right\rangle}
$$

The resulting VBG-model is a single input source and it is possible to apply the above procedure to identify the $m^{\text {th }}$ basis. Let it be $\bar{\omega}_{m}$. Therefore, the bases of $\Omega$ are:

$$
\left\{\begin{aligned}
\omega_{1} & =d y_{1} \\
\omega_{2} & =d y_{2} \\
& : \\
\omega_{m} & =\bar{\omega}_{m}+\sum_{i=1}^{m-1} p_{i}\left(\frac{d}{d t}\right) d y_{i}
\end{aligned}\right.
$$

The polynomials $\quad p_{i}\left(\frac{d}{d t}\right), i=1, \ldots, m-1, \quad$ with elements in $L\left[\frac{d}{d t}\right]$ must be chosen such that the above set of one forms is integrable.

Theorem (Fliess 1989)

A set of 1 -forms $\left(\omega_{1}, \ldots, \omega_{m}\right)$, of class $C^{1}$ and $\operatorname{rank}\left(\omega_{1}, \ldots, \omega_{m}\right)=m$ is integrable if and only if

$$
d \omega_{i} \wedge \omega_{1} \wedge \ldots \wedge \omega_{m}=0 \quad 1 \leq i \leq p
$$

$\Lambda$ stands for the skew tensor product

\section{REFERENCES}

Achir, A. 2005. Contribution à l'étude de la propriété de platitude sur des modèles Bond Graphs non linéaires. $\mathrm{PhD}$ Thesis, Université des Sciences et Technologies de Lille, France (December).

Achir, A., C. Sueur and G. Dauphin-Tanguy. 2005. "Bond graph and flatness based backstepping control of a salient permanent magnet synchronous motor" Journal of Systems and Control Engineering, Part I, ImechE, Professional Engineering Publishing, Number $I 7$ (November), pp. 461476.

Achir, A. and Sueur, C. 2005 "Non commutative ring bond graphs: application to flatness". International Conference on Bond Graph Modelling (ICBGM'05), WMC SCS (New Orleans, USA, January 23-27), F. E. Cellier, J. J Granda, editors, Simulation Series, vol. 37, n 1, pp. 59- 64.
Bertrand, J. M., Sueur, C. and G. Dauphin -Tanguy. 1997. "Bond graph modelling and geometric approach: input output decoupling with stability". IFAC Conference on Control of Industrial systems (Belford, France), pp. 317322.

Fliess, M. 1989. “Automatique et corps différentiels", Forum Math. 1, pp. 227-238.

Fliess, M. 1990. "Some basic structural properties of generalized linear systems". System and Control Letters 15, pp. 391-396.

Fliess, M., Lévine, J., Martin, Ph. and Rouchon, P. 1995. "Flatness and defect of nonlinear systems : introductory theory and examples". Int. J. of Control 61(6), pp. 13271361.

Fliess, M. 1998. "Some new interpretations of controllability and their practical implications". Proceedings of the IFAC Conference on System Structure and Control (Nantes, France), pp. 49-58.

Fossas, E., Franch, J. and Palau, A. 1998. "Flatness, tangent systems and flat outputs". Proceedings of the American Control Conference (Philadelphia, Pennsylvania), vol. 1, pp. 313-317.

Karnopp, D., Margolis, D. and Rosenberg, R. 1990. System Dynamics : A Unified Approach, New York, Wiley (2nd ed.). 